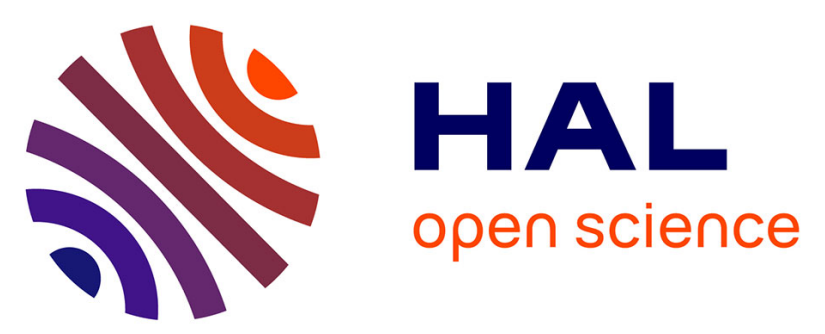

\title{
Draft genome sequence of a novel Bacillus glycinifermentans strain having antifungal and antibacterial properties
}

\author{
A. Karim, Olivier Poirot, A. Khatoon, M. Aurongzeb
}

\section{To cite this version:}

A. Karim, Olivier Poirot, A. Khatoon, M. Aurongzeb. Draft genome sequence of a novel Bacillus glycinifermentans strain having antifungal and antibacterial properties. Journal of Global Antimicrobial Resistance, 2019, 19, pp.308-310. 10.1016/j.jgar.2019.10.011 . hal-02409583

\section{HAL Id: hal-02409583 \\ https://hal-amu.archives-ouvertes.fr/hal-02409583}

Submitted on 13 Dec 2019

HAL is a multi-disciplinary open access archive for the deposit and dissemination of scientific research documents, whether they are published or not. The documents may come from teaching and research institutions in France or abroad, or from public or private research centers.
L'archive ouverte pluridisciplinaire HAL, est destinée au dépôt et à la diffusion de documents scientifiques de niveau recherche, publiés ou non, émanant des établissements d'enseignement et de recherche français ou étrangers, des laboratoires publics ou privés. 


\title{
Draft genome sequence of a novel Bacillus glycinifermentans strain having antifungal and antibacterial properties
}

\author{
A. Karim ${ }^{\mathrm{a}, *}$, O. Poirot ${ }^{\mathrm{b}}$, A. Khatoon ${ }^{\mathrm{a}}$, M. Aurongzeb ${ }^{\mathrm{a}}$ \\ a Jamil-Ur-Rahman Center for Genome Research, Dr Panjwani Center for Molecular Medicine and Drug Research (PCMD), International Center for Chemical \\ and Biological Sciences (ICCBS), University of Karachi, Karachi 75270, Pakistan \\ ${ }^{\mathrm{b}}$ Aix-Marseille Université, CNRS, Structural and Genomic Information Laboratory, UMR 7256 (IMM FR 3479), 163 Avenue de Luminy, Case 934, 13288, \\ Marseille Cedex 9, France
}

\begin{abstract}
Objectives: Bacillus spp. have been used as biocontrol agents against soilborne pathogens because they produce secondary metabolites that exhibit a wide range of antibacterial or antifungal properties. In this study, a novel strain of Bacillus glycinifermentans sp. (JRCGR-1) was identified and its genome was sequenced and annotated. The genome was explored for putative genes involved in antimicrobial activity. Methods: Whole-genome sequencing was performed on an Illumina NextSeq 500 platform. Read quality was checked by FastQC, paired-end reads were trimmed using Sickle, and de novo assembly was performed using SPAdes v.3.11.11. QUAST 5.02 was used to assess the quality of contigs and scaffolds. Finally, the assembled scaffolds were annotated by Prokka v.1.13. Genes involved in antimicrobial metabolite biosynthesis were predicted using antiSMASH. Virulence and antimicrobial resistance genes were predicted using BacWGSTdb and the Comprehensive Antibiotic Resistance Database (CARD), respectively.

Results: The genome of B. glycinifermentans JRCGR-1 was 4700692 bp in size with a G $+C$ content of $45.52 \%$. Final assembly of the genome resulted into 84 contigs and 83 scaffolds ( $>500$ bp length). Overall, the genome comprises 5174 genes, 32 tRNAs, 4 rRNAs, 1 tmRNA and 92 misc_RNAs. Eleven putative gene clusters responsible for antimicrobial metabolite biosynthesis were identified, including genes for biosynthesis of non-ribosomal lipopeptides and polyketides. Virulence and antimicrobial resistance genes were also identified in the genome.

Conclusion: The presence of antimicrobial resistance genes in the genome of $B$. glycinifermentans JRCGR-1

makes it a potential biocontrol agent against soilborne pathogens.
\end{abstract}

Soilborne pathogens are emerging as a global threat to agricultural products, and each year worldwide they cause huge economic losses. Several chemical-based fertilisers and pesticides are used to control soilborne pathogens. However, with long-term use these chemicals are hazardous to human health and cause environment pollution. An alternative to overcome this problem is the use of biocontrol agents [1]. Specifically, the genus Bacillus is a potent candidate for biocontrol agents against soilborne pathogens as members of this genus produce a wide range of secondary metabolites, hydrolytic enzymes and volatile organic compounds with antibacterial or antifungal properties.

Much work has been done to explore the biochemistry and physiology of Bacillus spp. Advancements in the field of whole- genome sequencing have given a new dimension to microbialbased products and processes.

In 2015, two bacterial isolates from cheonggukjang, a Korean fermented soybean paste food product, were found to comprise a novel Bacillus sp. named Bacillus glycinifermentans sp. nov. [2]. To date, only five B. glycinifermentans genome sequences are available in public databases, including three complete genomes (GenBank Assembly accession nos. GCA_900093775.1, GCA_002443095.1 and GCA_004103615.1) and two draft genomes (GenBank Assembly accession nos. LECV00000000.1 and LECW00000000.2). To the best of our knowledge, this is the first draft genome sequence of

B. glycinifermentans reported from Pakistan.

To study the genome of B. glycinifermentans strain JRCGR-1, the strain was first cultured on a nutrient agar plate. Colonies were then inoculated into $10 \mathrm{~mL}$ of Luria broth and were incubated at $37^{\circ} \mathrm{C}$ for $24 \mathrm{~h}$. The optical density was adjusted to a McFarland 
standard of $3-4 \mathrm{~nm}$. Following incubation, bacterial cells were collected by centrifugation $(3000 \times g$ for $15 \mathrm{~min})$. The supernatant was removed without disturbing the sediment and the DNA was extracted using a commercial DNA extraction kit (QIAamp ${ }^{\circledR}$ DNA Mini Kit; QIAGEN, Hilden, Germany). A Qubit ${ }^{\mathrm{TM}}$ dsDNA BR Assay kit (Invitrogen; Thermo Fisher Scientific, Eugen, OR, USA) was used to calculate the amount of DNA using a Qubit ${ }^{\mathbb{R}} 2.0$ fluorometer
(Invitrogen) according to the manufacturer's instructions. A Nextera XT DNA Library Kit (Illumina Inc., San Diego, CA, USA) was used to construct a paired-end library, and sequencing was performed on a NextSeq 500 platform (Illumina Inc.) in paired-end read mode. The quality of the reads was checked using FastQC software. Paired-end reads were then trimmed using Sickle and de novo assembly was performed using SPAdes v.3.11.11 into contigs and scaffolds (base

Table 1

Secondary metabolite profile of six Bacillus glycinifermentans strains

\begin{tabular}{|c|c|c|}
\hline Strain/metabolite type & Most similar known cluster & Similarity (\%) \\
\hline \multicolumn{3}{|l|}{ B. glycinifermentans GO-13 } \\
\hline Lanthipeptide & Geobacillin II & 50 \\
\hline NRPS & Lichenysin & 100 \\
\hline T3PKS & - & - \\
\hline NRPS & Fengycin & 26 \\
\hline Siderophore & - & - \\
\hline$\beta$-Lactone & Fengycin & 53 \\
\hline Lasso peptide & - & - \\
\hline NRPS & Bacillibactin & 53 \\
\hline NRPs, T1PKS, terpene & Paenibacterin & 60 \\
\hline \multicolumn{3}{|l|}{ B. glycinifermentans $\mathrm{KJ}-17$} \\
\hline Thiopeptide, bacteriocin & Butirosin & 7 \\
\hline NRPS, terpene, T1PKS & Paenibacterin & 60 \\
\hline Lasso peptide & - & - \\
\hline T3PKS & - & - \\
\hline Lanthipeptide & Geobacillin & 50 \\
\hline Siderophore & - & - \\
\hline NRPS & Fengycin & 33 \\
\hline NRPS & Bacillibactin & 46 \\
\hline NRPS & Lichenysin & 100 \\
\hline$\beta$-Lactone & Fengycin & 53 \\
\hline \multicolumn{3}{|l|}{ B. glycinifermentans BGLY } \\
\hline Sactipeptide & Sporulation killing factor SkfA & 85 \\
\hline NRPS & Lichenysin & 100 \\
\hline Thiopeptide, bacteriocin & Butirosin & 7 \\
\hline Siderophore & - & - \\
\hline$\beta$-Lactone & Fengycin & 53 \\
\hline Terpene & - & - \\
\hline T3PKS & - & - \\
\hline NRPS & Bacitracin & 88 \\
\hline NRPS & Bacillibactin & 53 \\
\hline \multicolumn{3}{|l|}{ B. glycinifermentans KBNO06PO3352 } \\
\hline NRPS & Fengycin & 26 \\
\hline$\beta$-Lactone & Fengycin & 53 \\
\hline NRPS, T1PKS, terpene & Paenibacterin & 60 \\
\hline T3PKS & - & - \\
\hline NRPS & - & - \\
\hline Lasso peptide & - & - \\
\hline Lanthipeptide & Geobacillin & 50 \\
\hline NRPS & Lichenysin & 100 \\
\hline Thiopeptide, bacteriocin & Butirosin & 7 \\
\hline \multicolumn{3}{|l|}{ Siderophore } \\
\hline \multicolumn{3}{|l|}{ B. glycinifermentans SRCM103574 } \\
\hline Sactipeptide (head-to-tail cyclised peptide) & Sporulation killing factor SkfA & 85 \\
\hline NRPS & Lichenysin & 100 \\
\hline Thiopeptide, bacteriocin & Butirosin & 7 \\
\hline Siderophore & - & - \\
\hline$\beta$-Lactone & Fengycin & 53 \\
\hline Terpene & - & - \\
\hline T3PKS & - & - \\
\hline NRPS & Bacitracin & 88 \\
\hline NRPS & Bacillibactin & 53 \\
\hline \multicolumn{3}{|l|}{ B. glycinifermentans JRCGR-1 } \\
\hline T3PKS & - & - \\
\hline Terpene & - & - \\
\hline$\beta$-Lactone & Fengycin & 53 \\
\hline NRPS & Lichenysin & 100 \\
\hline Head-to-tail & Sporulation killing factor SkfA & 71 \\
\hline Siderophore & - & - \\
\hline NRPS & Bacillibactin & 53 \\
\hline NRPS & Bacitracin & 66 \\
\hline Bacteriocin & - & - \\
\hline NRPS & - & - \\
\hline NRPS & Bacitracin & 33 \\
\hline
\end{tabular}

NRPS, non-ribosomal peptide synthetase. 
quality scores $>$ Q20, $k=39$ ). The quality of contigs and scaffolds was evaluated by QUAST 5.02. The assembled contigs were annotated using Prokka v.1.13 [>500 bp; e-value cut-off default (10_6)] for rapid annotation of prokaryotic genomes. tRNAscan-SE was used for the prediction of tRNA genes. The plasmid was assembled using plasmidSPAdes (base quality scores $>$ Q20, $k=55$ ), and gene annotation was done using Prokka v.1.13 [>500 bp; e-value cutoff default (10_6)]. RNAmmer and Barrnap were used to identify RNA genes. Putative genes involved in antimicrobial metabolite biosynthesis were predicted using antiSMASH v.5.0.0rc1. Virulence genes were predicted using the BacWGSTdb service (http://bacdb. org/BacWGSTdb/index.php). Antimicrobial resistance genes were predicted using the Comprehensive Antibiotic Resistance Database (CARD) (https://card.mcmaster.ca/home).

A total of 4851845 paired-end reads $(2 \times 76$-bp $)$ were generated with $32 \times$ coverage for strain JRCGR-1. The genome assembly contained 4700692 bp and with an average $G+C$ content of $45.52 \%$. The final assembly contains 84 contigs and 83 scaffolds ( $>500$ bp length, $N_{50}$ of $135232 \mathrm{bp}$ ). The maximum contig size was $384553 \mathrm{bp}$. Overall, the genome comprises 5174 genes, 32 tRNAs, 4 rRNAs, 1 tmRNA and 92 misc_RNAs. The plasmid was assembled into 37 scaffolds using plasmidSPAdes. Other features of the plasmid include a size of 1113267 bp, 27 tRNAs, 4 rRNA, 1366 genes, 21 misc_RNAs and 1314 CDS (coding sequences).

Genes involved in antimicrobial metabolite biosynthesis were predicted using antiSMASH. Draft genome analysis of strain JRCGR1 revealed 11 putative gene clusters responsible for antimicrobial metabolite biosynthesis, among which five encode non-ribosomal peptide synthetases (NRPS) (for biosynthesis of one fengycin, one lichenysin, one bacillibactin and two bacitracins) and one related to terpene and $\beta$-lactone biosynthesis. Siderophores (bacillibactin) produced by bacteria are involved in inhibition of phytopathogen growth by depriving them of essential iron [3].

Supplementary Figs. S1 and S2 show two putative gene clusters of strain JRCGR-1 (label as query sequence) involved in biosynthesis of secondary metabolites. These genes have similarities with non-ribosomal lipopeptides (lichenysin, surfactin, fengycin, mycosubtilin and plipastatin) and polyketides (basiliskamides). Supplementary Fig. S1(A) shows the first putative gene cluster of strain JRCGR-1 involved in secondary metabolite biosynthesis and its similarity to lichenysin (100\%), surfactin (47\%) and basiliskamides (9\%). Comparison of this gene against other Bacillus spp. shows $94 \%$ similarity with B. glycinifermentans strain GO-13, B. glycinifermentans strain KBNO06PO3352 and B. glycinifermentans strain BGLY [Supplementary Fig. S1(B)]. Similarly, the second putative gene cluster presented similarities with fengycin (53\% similarity), mycosubtilin (40\% similarity) and plipastatin (30\% similarity) [Supplementary Fig. S2(A)]. Comparison of this predicted gene cluster of strain JRCGR-1 with other Bacillus spp. showed similarity between $45-50 \%$ for seven Bacillus paralicheniformis sp. and $46 \%$ gene similarity for Bacillus licheniformis strain B4123 [Fig. S2(B)]. Secondary metabolites for five other strains of B. glycinifermentans were also predicted using antiSMASH. The finding reveals that fengycin and lichenysin were present as core genes, i.e. they were present in all six strains. A putative gene for bacillibactin was absent in B. glycinifermentans strain KBNO06PO3352. Sporulation killing factor SkfA was present in B. glycinifermentans strain JRCGR1 and B. glycinifermentans strain SRCM103574. Details of the secondary metabolite profile of six strains of B. glycinifermentans sp. are given in Table 1 . The presence of these NRPS genes can be linked with the biocontrol potential of bacteria [4]. Several gene cluster were also identified that can suppress the growth of Grampositive bacteria by producing synthetases (PKS), e.g. oleandomycin, $\beta$-lactamase, tetracenomycin and tetracycline. Putative bsIA $(y u a B)$ and $\operatorname{tas} A$ genes associated with the production of biofilm matrix and fungal cell-wall-degrading enzymes, respectively, were also identified. In the genomes of six strains of $B$. glycinifermentans sp., two antimicrobial resistance genes (bcrA and Escherichia coli ampC1 $\beta$-lactamase) were present as accessory genes. These genes were present in three strains of $B$. licheniformis, including SRCM103574, BGLY and JRCGR-1. The bcrA gene is an ATP-binding cassette $(A B C)$ transporter found mostly in $B$. licheniformis sp. that confers bacitracin resistance [5]. The E. coli AmpC1 $\beta$-lactamase resistance mechanism is based on antibiotic inactivation, whereas for BcrA the resistance mechanism involves antibiotic efflux. In the genomes of strains KBNO06PO3352 and GO-13, only E. coli ampC1 $\beta$-lactamase was predicted. On the other hand, strain $\mathrm{KJ}-17$ lacks all of these genes. We also identified virulence genes in the strain JRCGR-1 genome, including $\operatorname{capA}, \operatorname{capB}, \operatorname{capC}$ and hlyIII, with similarities of $83.25 \%, 89.30 \%, 88 \%$ and $82.26 \%$, respectively.

The aforementioned findings reveal that B. glycinifermentans JRCGR-1 might be a potential candidate as a biological control agent against plant soilborne diseases.

\section{GenBank accession no}

The draft genome sequence of B. glycinifermentans JRCGR-1 was submitted to the NCBI database with accession no. VHPY0000000.

\section{Funding}

This study was supported by funds from the International Center for Chemical and Biological Sciences (ICCBS), University of Karachi (Karachi, Pakistan), Campus France and Embassy of France in Pakistan.

\section{Competing interests}

None declared.

\section{Ethical approval}

Not required.

\section{Acknowledgments}

The authors would like to thank Matthieu Legendre, Chantal Abergel, Sébastien Santini and Sébastien Nin [Aix-Marseille Université, CNRS, Structural and Genomic Information Laboratory, UMR 7256 (IMM FR 3479)] and Georges Massiot [University of Reims, Reims, France] for their valuable support.

\section{Appendix A. Supplementary data}

Supplementary material related to this article can be found, in the online version, at doi:https://doi.org/10.1016/j.jgar.2019.10.011.

\section{References}

[1] Cook RJ. Making greater use of introduced microorganisms for biological control of plant pathogens. Annu Rev Phytopathol 1993;31:53-80.

[2] Kim S-J, Dunlap CA, Kwon S-W, Rooney AP. Bacillus glycinifermentans sp. nov., isolated from fermented soybean paste. Int J Syst Evol Microbiol 2015;65:3586-90.

[3] Chen XH, Koumoutsi A, Scholz R, Eisenreich A, Schneider K, Heinemeyer I, et al. Comparative analysis of the complete genome sequence of the plant growthpromoting bacterium Bacillus amyloliquefaciens FZB42. Nat Biotechnol 2007;25:1007-14.

[4] Zhang S, Jiang W, Li J, Meng L, Cao X, Hu J, et al. Whole genome shotgun sequence of Bacillus amyloliquefaciens TF28, a biocontrol entophytic bacterium. Stand Genomic Sci 2016;11:73.

[5] Podlesek Z, Comino A, Herzog-Velikonja B, Žgur-Bertok D, Komel R, Grabnar M. Bacillus licheniformis bacitracin-resistance $A B C$ transporter: relationship to mammalian multidrug resistance. Mol Biol 1995;16:969-76. 